\title{
Topological events on wave dislocation lines: birth and death of loops, and reconnection
}

\author{
M V Berry ${ }^{1}$ and M R Dennis ${ }^{2}$ \\ ${ }^{1}$ H H Wills Physics Laboratory, Tyndall Avenue, Bristol BS8 1TL, UK \\ ${ }^{2}$ School of Mathematics, University of Southampton, Highfield, Southampton SO17 1BJ, UK
}

Received 6 October 2006

Published 6 December 2006

Online at stacks.iop.org/JPhysA/40/65

\begin{abstract}
In three-dimensional space, a wave dislocation, that is, a quantized (optical) vortex or phase singularity, is a line zero of a complex scalar wavefunction. As a 'time' parameter varies, the topology of the vortex can change by encounter with a line of vanishing vorticity (curl of the current associated with the wavefunction). An isolated critical point of the field intensity, sliding along the zero-vorticity line like a bead on a wire, meets the vortex as it encounters the line, and so participates in the singular event. Local expansion and gauge and coordinate transformations show that the vortex topology can change generically by the appearance or disappearance of a loop, or by the reconnection of branches of a pair of hyperbolas.
\end{abstract}

PACS numbers: $02.40 . \mathrm{Xx}, 03.65 . \mathrm{Vf}, 03.75 . \mathrm{Lm}$, 42.25.Fx, 67.40.Vs

\section{Introduction}

Lines of phase singularity are important morphological elements of complex scalar fields. When the field represents a wave (for example a linearly polarized optical field), phase singularities are also known as wave dislocations, wave (or optical) vortices, and nodal lines. They have been studied extensively, both theoretically and experimentally [1-4]. In quantum waves, they are also referred to as quantized vortex lines, and have important physical consequences $[5,6]$. Unlike their counterparts in fluid mechanics, the location of these wave vortices is determined purely by the vanishing of the complex wavefunction, so that wave vortices are places where the phase is undefined. In three-dimensional space, the vortex lines display a variety of geometries, and can be curved, linked or knotted [7-10], in ways at most only weakly constrained by equations (linear or not) that the waves satisfy.

Here we will investigate phase singularity lines in three-dimensional space, whose geometry depends on a parameter that we will call 'time'. Our aim is to establish the generic (that is, structurally stable) events in which phase singularities in space can meet and change their topology, or spontaneously appear and disappear. Some of our conclusions have appeared 
in previous studies of controlled creation and reconnection events in optical vortices [11, 12], associated with knots, links and braids $[8,13]$, and the development of diffraction catastrophes [14] and other three-dimensional situations where a parameter varies [15, 16]. Reconnections have also been studied for vortices in superfluids [17], in the Schrödinger equation (both linear [18] and nonlinear [19]), and in cosmic strings [20]. However, the subject deserves a closer look, because general statements and formulas, as well as a connection we uncover between phase and intensity singularities, have been lacking.

The fact that vortices are lines follows from a familiar codimension argument: as nodal lines in the complex wavefield, they occur on the lines of intersection of the zero contour surfaces of the real and imaginary parts of the field (i.e. the vortices have codimension 2). The events we study here, in which the vortices change topology, have codimension 4-two to be at a node (zero surfaces intersect), and two more so that the tangent planes to the zero surfaces are parallel, so the surfaces touch. The details will be described in section 3 .

Here we will be studying topological changes of vortex lines in three dimensions. Interactions between vortex points in the plane are much simpler: generically, points can appear or annihilate in pairs of opposite strength, these events occurring in the presence of phase saddles [21]. If the plane being considered is an observation screen $\{x, y\}$ in threedimensional space $\{x, y, z\}$ (for example in paraxial optics), interactions of points on the screen as $z$ varies can give the illusion of a three-dimensional interaction, when all that is really happening is a tangency between the screen and a smoothly curved vortex line in space [22].

\section{Participating singularities}

Let the waves $\psi$ that we will consider, expressed in terms of their modulus, phase, and real and imaginary parts, be written as

$$
\psi(\boldsymbol{r}, t)=\rho(\boldsymbol{r}, t) \exp \{\mathrm{i} \chi(\boldsymbol{r}, t)\}=\xi(\boldsymbol{r}, t)+\mathrm{i} \eta(\boldsymbol{r}, t) \quad(\boldsymbol{r}=\{x, y, z\}) .
$$

The vortices, which have codimension 2 and so are lines in space, satisfy

$$
\text { vortex lines: } \psi=0 \Rightarrow \xi=\eta=0 \text {. }
$$

They are quantized: in a loop around the vortex line, the phase changes by $2 \pi$ (quantized vortices of higher strength have a higher codimension, and are not considered here). Associated with the phase are two vectors. First is the current $j$, whose streamlines (orthogonal to the wavefronts $\chi=$ constant) wind around the vortices [23, 24]:

$$
j=\operatorname{Im} \psi^{*} \nabla \psi=\rho^{2} \nabla \chi .
$$

Second is the vorticity

$$
\boldsymbol{\Omega}=\frac{1}{2} \nabla \times j=\frac{1}{2} \operatorname{Im}\left(\nabla \psi^{*} \times \nabla \psi\right)=\nabla \xi \times \nabla \eta .
$$

This vector exists everywhere, and in particular on a vortex line, whose tangent at $r$ is parallel to $\Omega(\boldsymbol{r})$ (because vortices are intersections of the surfaces $\xi=0, \eta=0$ ).

Loci of vanishing $\Omega$ (irrotational lines) play an important role in the life of a vortex line, because if $\Omega=0$ on a vortex line, its tangent direction is undefined. The loci are

$$
\Omega=0 \text {, i.e. } \nabla \xi \text { and } \nabla \eta \text { parallel (or antiparallel). }
$$

Thus where $\Omega=0$ the complex vector $\nabla \psi$ is a real vector (in the common direction of $\nabla \xi$ and $\nabla \eta$ ) multiplied by a phase factor. The loci are lines, notwithstanding the three conditions corresponding to each component of $\Omega$, because these are not independent: from (4), $\Omega=$ 0 corresponds to the vectors $\nabla \xi$ and $\nabla \eta$ being parallel, which in three-dimensional space is 
a codimension 2 requirement. Usually the $\Omega=0$ lines and the vortices are distinct, but, as is intuitively plausible and will be shown in the next section, encounter is possible as time varies. We will show that at such events (codimension 4 in total), the topology of the vortex line changes, in one of two possible ways.

First we note a connection, important in what follows, between singularities of phase and the intensity

$$
I=|\psi|^{2}=\rho^{2}
$$

Consider the loci of vanishing $\nabla I$. These fall into two classes. First are the vortices: lines (codimension 2) where $\rho$ vanishes, across which $\rho^{2}$ has a smooth minimum. In addition, there are points (codimension 3 ) where $I$ does not vanish:

$$
\begin{aligned}
& \text { intensity critical points: } \\
& \text { isolated points where } \nabla I=0 \text {, i.e. } \xi \nabla \xi+\eta \nabla \eta=0 \text { and } I \neq 0 \text {. }
\end{aligned}
$$

At intensity critical points, $\nabla \xi$ and $\nabla \eta$ must be parallel or antiparallel, so these points lie on the $\Omega=0$ lines. Over time, the intensity critical points move along the $\Omega=0$ lines like beads sliding on a wire, and the $\Omega=0$ lines themselves can move. In the next section, we will show that as a vortex meets an $\Omega=0$ line at a topology-changing event, an isolated intensity critical point participates in the encounter by arriving at the same instant.

It is not hard to confirm that vortex lines and isolated intensity critical points are invariant under arbitrary gauge transformations (multiplication by a smooth, possibly coordinate- and time-dependent phase factor, that is, a rotation in the $\xi, \eta$ plane), and arbitrary smooth linear or nonlinear coordinate transformations. The $\Omega=0$ lines, on the other hand, generally deform under a gauge transformation, though they are pinned at the isolated intensity critical points. (If a constant is added to $\psi$, the vortices and intensity critical points move, but the $\Omega=0$ lines do not; this property was used [8] to tune reconnections in knotting vortices.)

\section{Neighbourhood of the topology-changing events}

Consider an $\Omega=0$ line passing through the origin at some instant, so that $\nabla \xi$ and $\nabla \eta$ are parallel. If a vortex line passes though the origin at the same time, $\psi$ must have the local form

$$
\psi=\boldsymbol{a} \cdot \boldsymbol{r}+\frac{1}{2} \boldsymbol{r} \cdot \mathrm{A} \cdot \boldsymbol{r}+\cdots
$$

where $\boldsymbol{a}$ is a vector whose real and imaginary parts are parallel (i.e. $\boldsymbol{a}$ is a real vector times a phase factor, so $\boldsymbol{a}^{*} \times \boldsymbol{a}=0$ ), and $\mathrm{A}$ is a complex $3 \times 3$ symmetric matrix. At small non-zero times, the vortex and the $\Omega=0$ line will typically no longer coincide, and neither will pass through the origin. However, by choosing a moving coordinate frame the $\Omega=0$ line can be made to continue to pass through the origin. But $\psi$ will no longer vanish at $r=0$.

At first it seems as though a complex 'time' parameter, $\tau$ say, is required to unfold the singularity, that is

$$
\psi=\tau+\boldsymbol{a} \cdot \boldsymbol{r}+\frac{1}{2} \boldsymbol{r} \cdot \mathrm{A} \cdot \boldsymbol{r}+\cdots .
$$

This would correspond to the two real parameters $\operatorname{Re} \tau$ and $\operatorname{Im} \tau$. Nevertheless, we now show that the events we are considering have real codimension 4: space $(\boldsymbol{r})$ plus just one real time parameter. Referring to figure 1 , we consider values of $\psi$ at points on the $\Omega=0$ line, in the complex $\xi, \eta$ plane. When the $\Omega=0$ line intersects a dislocation line at the coordinate origin, its image in the $\psi$ plane crosses O. For times close to this intersection, there will be a point $\mathrm{P}$ closest to $\mathrm{O}$, where it is obvious that $I=|\psi|^{2}=\xi^{2}+\eta^{2}$ is stationary with respect to displacements along the $\Omega=0$ line, that is

$$
\boldsymbol{d} \cdot \nabla I=\boldsymbol{d} \cdot \boldsymbol{e}|\nabla \xi|(\xi+c \eta)=0,
$$




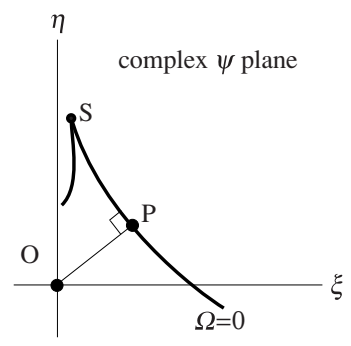

Figure 1. Complex $\psi$ plane for a time near a topology-changing event. The origin $\mathrm{O}$ corresponds to the dislocation, the thick curve is the image of the $\Omega=0$ line (5), on which lie the point $\mathrm{P}$ corresponding to an isolated intensity critical point (7), and the cusp point $\mathrm{S}$ (irrelevant to the topology-changing event) corresponding to a place where the $\Omega=0$ line is perpendicular to the common direction of $\nabla \xi$ and $\nabla \eta$.

where $\boldsymbol{d}$ is the direction of the $\Omega=0$ line, and $e$ and $c$ are defined by

$$
\nabla \eta=c \nabla \xi=c|\nabla \xi| e .
$$

The condition (10) corresponding to $\mathrm{P}$ can be satisfied either by $\nabla I=0$ (i.e. $\xi+$ $c \eta=0$ ) or by $\boldsymbol{d} \cdot e=0$. We ignore points $\mathrm{S}$ where $\boldsymbol{d} \cdot e=0$, because when $\nabla \xi$ and $\nabla \eta$ are perpendicular to the direction of the $\Omega=0$ line neither $\xi$ nor $\eta$ is changing, and $\mathrm{S}$ corresponds to a special (cusp) point, unrelated to $\mathrm{O}$, requiring two parameters to encounter $\mathrm{O}$ (we will return to the $\boldsymbol{d} \cdot e=0$ points later). On the other hand, one parameter suffices to make the image curve encounter $\mathrm{O}$, so at the corresponding $\mathrm{P}$, where the curve is smooth, we must have $\xi+c \eta=0$. This implies the vanishing of all components of $\nabla I$, not just the component along the $\Omega=0$ line. Thus we have justified the assertion that an isolated critical point must participate in the topology-changing event.

It is convenient to choose $\tau$ real in a gauge where $\boldsymbol{a}$ is imaginary, and orient coordinate axes such that $\boldsymbol{a}$ points in the $z$ direction. Thus without loss of generality (9) can be replaced by

$$
\psi=t+\mathrm{i} a z+\frac{1}{2} \boldsymbol{r} \cdot \mathrm{A} \cdot \boldsymbol{r}+\cdots,
$$

where $t$ and $a$ are real and again A is a complex $3 \times 3$ symmetric matrix.

The vortex lines at time $t$ are conveniently determined by separating the components of $r$ perpendicular and parallel to $a$, so

$$
\begin{aligned}
& t+\frac{1}{2} \boldsymbol{R} \cdot\left(\operatorname{Re} \mathrm{A}_{\perp}\right) \cdot \boldsymbol{R}+z \operatorname{Re} \boldsymbol{b} \cdot \boldsymbol{R}+\frac{1}{2} z^{2} \operatorname{Re} A_{33}=0 \\
& a z+\frac{1}{2} \boldsymbol{R} \cdot\left(\operatorname{Im} \mathrm{A}_{\perp}\right) \cdot \boldsymbol{R}+z \operatorname{Im} \boldsymbol{b} \cdot \boldsymbol{R}+\frac{1}{2} z^{2} \operatorname{Im} A_{33}=0,
\end{aligned}
$$

where

$$
\boldsymbol{r}=\{\boldsymbol{R}, z\}, \quad \boldsymbol{R}=\{x, y\}, \quad \mathrm{A}_{\perp}=\left(\begin{array}{cc}
A_{11} & A_{12} \\
A_{12} & A_{22}
\end{array}\right), \quad \boldsymbol{b}=\left\{A_{13}, A_{23}\right\} .
$$

From the second equation in (13), representing the $\eta=0$ surface, the vortices lie on the fixed surface with height

$$
z=-\frac{\boldsymbol{R} \cdot\left(\operatorname{Im} \mathrm{A}_{\perp}\right) \cdot \boldsymbol{R}}{2 a}+\cdots,
$$

where ... represents terms cubic and higher in $\boldsymbol{R}$. The first equation in (13), representing the $\xi=0$ surface, gives the projection of the vortices onto the plane $z=0$ :

$$
\boldsymbol{R} \cdot\left(\operatorname{Re} \mathrm{A}_{\perp}\right) \cdot \boldsymbol{R}=-2 t .
$$



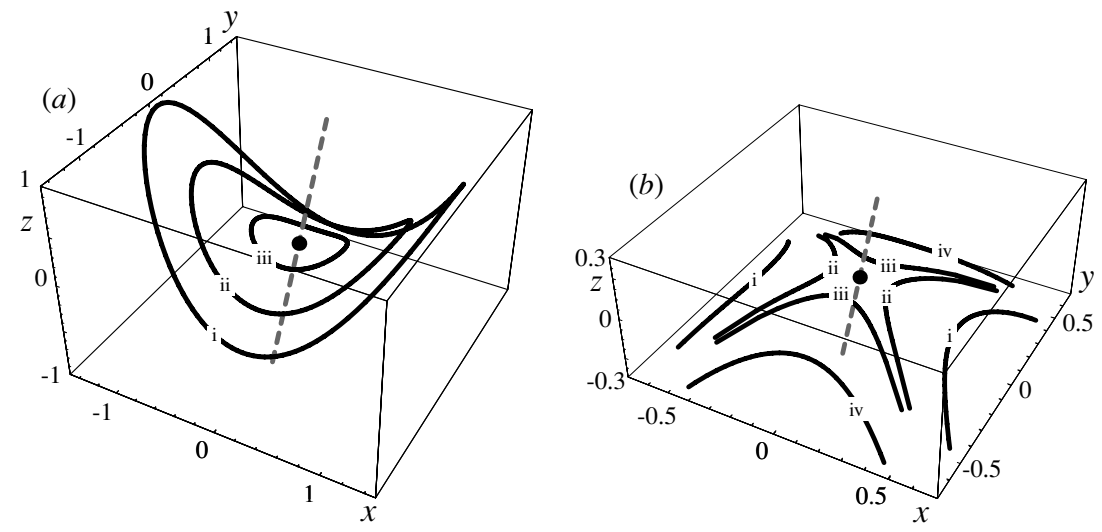

Figure 2. Topology-changing events. (a) Vortex loop shrinking and vanishing, for $\left(A_{11}=1-\mathrm{i}\right.$, $A_{22}=2+\mathrm{i}, A_{12}=A_{13}=0, A_{23}=-1, A_{33}$ arbitrary) and times i: $t=-1$; ii: $t=-0.5$; iii: $t=-0.1$; dashes denote the $\Omega=0$ line, and the black dot is the isolated intensity critical point. (b) As (a), for $\left(A_{11}=1+\mathrm{i}, A_{22}=-1+\mathrm{i}, A_{12}=A_{13}=0, A_{23}=0.5, A_{33}\right.$ arbitrary) and times i: $t=-0.1$; ii: $t=-0.01$; iii: $t=+0.01$; iv: $t=+0.1$.

In the gauge corresponding to (12), the vortex geometry is determined by the gaussian curvature of the $\xi=0$ surface, that is by $\operatorname{det} \operatorname{Re} A_{\perp}$. If $\operatorname{det} \operatorname{Re} A_{\perp}>0$, (16) represents an elliptical vortex loop shrinking to a point and vanishing as $t \rightarrow 0$ from below/above, if the eigenvalues of $\operatorname{Re} A_{\perp}$ are both positive/negative. If $\operatorname{det} \operatorname{Re} A_{\perp}<0$, (16) represents a pair of hyperbolas whose branches reconnect at $t=0$, when they degenerate into a cross. These are the two stable possibilities for vortex topology change, illustrated in figure 2 .

Equation (14) shows that in general the vortex is not planar, but flattens near the singularity. Using polar coordinates $\boldsymbol{R}=\{R, \phi\}$, we can combine (15) and (16) into

$$
\begin{aligned}
\frac{a z}{t} & =\frac{\operatorname{Im} A_{11} \cos ^{2} \phi+2 \operatorname{Im} A_{12} \sin \phi \cos \phi+\operatorname{Im} A_{22} \sin ^{2} \phi}{\operatorname{Re} A_{11} \cos ^{2} \phi+2 \operatorname{Re} A_{12} \sin \phi \cos \phi+\operatorname{Re} A_{22} \sin ^{2} \phi} \\
& =\tan \arg \boldsymbol{R} \cdot \mathbf{A}_{\perp} \cdot \boldsymbol{R},
\end{aligned}
$$

showing that each point on the vortex approaches the $\boldsymbol{R}$ plane at a constant speed, which in the hyperbolic case is infinitely fast at the asymptotes.

From

$$
\nabla \xi=\operatorname{Re} A \cdot r, \quad \nabla \eta=a e_{z}+\operatorname{ImA} \cdot \boldsymbol{r},
$$

it follows that the local form of the $\Omega=0$ line, where $\nabla \xi$ and $\nabla \eta$ are parallel, is determined by

$$
\left(a e_{z}+\operatorname{Im} \mathrm{A} \cdot \boldsymbol{r}\right) \times \operatorname{Re} \mathrm{A} \cdot \boldsymbol{r}=0 .
$$

In the appendix we give the general solution of this equation, and show that near the origin the form of the $\Omega=0$ line, parameterized by $z$, is

$$
\boldsymbol{R}(z)=z \frac{\left[(\operatorname{Re} \mathrm{A})^{-1} \cdot e_{z}\right]_{\perp}}{e_{z} \cdot(\operatorname{Re} \mathrm{A})^{-1} \cdot e_{z}}=-z\left\{\frac{\operatorname{Re} A_{13}}{\operatorname{Re} A_{11}}, \frac{\operatorname{Re} A_{23}}{\operatorname{Re} A_{22}}\right\},
$$

where in the last member we have chosen coordinates in which $A_{\perp}$ is diagonal. If the denominator of (20) vanishes, the $\Omega=0$ line is perpendicular to the $z$ direction, corresponding to the situation $\boldsymbol{d} \cdot \boldsymbol{e}=0$ discussed after (11); this is the critical case $\operatorname{det} \operatorname{Re} \mathrm{A}_{\perp}=0$, of a transition between the loop and reconnection topology-changing events. 
The single intensity critical point determined by (7) is fixed at the origin $r=0$. This follows directly from (12), which shows that when $t \neq 0$ the intensity $|\psi|^{2}$ has a quadratic critical point at $\boldsymbol{r}=0$; as previously argued, at the instant $t=0$ of the singular event, the vortex collides with the critical point, which becomes degenerate (locally cubic).

The general picture we have derived is of a vortex loop shrinking onto an isolated intensity critical point on an $\Omega=0$ line, or two vortex lines crossing at an isolated intensity critical point on an $\Omega=0$ line. Particular waves displaying both types of behaviour have been found before. The wave [1]

$$
\psi=\left[\frac{1}{2} k^{2} R^{2}+\mathrm{i} k z+\mathrm{i}(A-\mathrm{i} B)(k z-\omega t)\right] \exp \{\mathrm{i}(k z-\omega t)\}
$$

is an exact solution of the dispersionless equation for waves with speed $\omega / k$. After gauging away the irrelevant phase factor, and using dimensionless variables, it can be written as

$$
\xi=-B(t-z)+\frac{1}{2} R^{2}, \quad \eta=z(A+1)-A t .
$$

This does not have the form (12) but can easily be transformed into it. The wave represents the vortex loop

$$
R^{2}=-\frac{2 B t}{A+1}, \quad z=\frac{A t}{A+1},
$$

corresponding to the boundary of an expanding hole in a punctured wavefront, or the healing of a circular tear in a wavefront, or the birth or disppearance of a disk of wavefront, depending on the values of $A$ and $B$. The $\Omega=0$ line is the $z$ axis, along which the isolated critical point moves according to

$$
z=\frac{B^{2}+A(A+1)}{A^{2}+(A+1)^{2}} t
$$

Note that the loop and the isolated critical points move along $z$ at different speeds, but coincide at the singular event $t=0, r=0$.

As an example of the hyperbolic case, we previously [8] gave

$$
\psi=t+\mathrm{i} z-\frac{1}{2}\left(x^{2}+y^{2}\right)+\frac{x y}{\sin 2 \gamma} .
$$

This is an exact solution of both the Helmholtz and paraxial time-independent wave equations, in the form (12) with $A_{11}=A_{22}=-A_{12}=-1$, with $t$ playing the role of the 'time' parameter unfolding the singularity. In this case $\operatorname{Im} A=0$, so according to (15) the hyperbolic vortices are flat.

\section{Analogies with polarization singularities}

We are concerned here with complex scalar waves, but our analysis exhibits several analogies with polarization optics, which involves complex vector waves of the form

$$
\boldsymbol{E}(\boldsymbol{r}, t)=\boldsymbol{P}(\boldsymbol{r}, t)+\mathrm{i} \boldsymbol{Q}(\boldsymbol{r}, t) .
$$

The polarization singularities of the field $\boldsymbol{E}$ are the L and C lines [25], on which the light is respectively linearly and circularly polarized. They are defined [26] by

$$
\text { L line: } \operatorname{Im} \boldsymbol{E}^{*} \times \boldsymbol{E}=2 \boldsymbol{P} \times \boldsymbol{Q}=0 ; \quad \text { C line: } \quad \boldsymbol{E} \cdot \boldsymbol{E}=0 .
$$

In the first analogy, we define from $\psi$ the vector field

$$
\boldsymbol{E}_{1}=\nabla \psi
$$


At a dislocation $\psi=0$, the anisotropy ellipse $[23,27]$ is the same as the polarization ellipse $[25,26,28]$ of $\boldsymbol{E}_{1}$, with principal axes interchanged. From (4) and (5), the analogy is

$$
\text { L line of } \boldsymbol{E}_{1} \quad \Leftrightarrow \quad \Omega=0 \text { line of } \psi \text {. }
$$

In planes transverse to the $\Omega=0$ line, $\Omega$ exhibits the characteristic singularities of a gradient vector field: circulations (when the vortices are locally loops) and saddles (when the vortices are locally hyperbolas), depending on the local L line index [25, 29]. (In general non-gradient polarization fields $\boldsymbol{E}$, source, sink and spiral singularities can also occur.) Unlike $\Omega=0$ lines, which, as we have noted, deform under gauge transformations $\psi(\boldsymbol{r}) \rightarrow \exp \{\mathrm{i \chi}(\boldsymbol{r})\} \psi(\boldsymbol{r})$, L lines are invariant under the different gauge transformations $\boldsymbol{E}(\boldsymbol{r}) \rightarrow \exp \{\mathrm{i} \chi(\boldsymbol{r})\} \boldsymbol{E}(\boldsymbol{r})$ appropriate for general complex vector fields. The S points discussed in the previous section, where $\boldsymbol{d} \cdot \boldsymbol{e}=0$ (so the $\Omega=0$ line is perpendicular to $\nabla \xi$ and $\nabla \eta$ ), are points where the index of the L line switches sign [25]. There is a general formula [26] for the direction of an L line; this can be applied to the field $\boldsymbol{E}_{1}$ generated by (12), and gives an alternative derivation of the local form (20) of the $\Omega=0$ line.

In the second analogy, we start from $\boldsymbol{E}$ and define the scalar field

$$
\psi_{1}=\boldsymbol{E} \cdot \boldsymbol{E},
$$

whence (25) shows that [26]

$$
\text { C line of } \boldsymbol{E} \quad \Leftrightarrow \quad \text { vortex line of } \psi_{1} \text {. }
$$

It follows that the topology-changing events of these $\mathrm{C}$ lines occur when they encounter $\mathrm{L}$ lines of the associated vector field

$$
\boldsymbol{E}_{2}=\nabla(\boldsymbol{E} \cdot \boldsymbol{E})=2(\boldsymbol{E} \cdot \nabla) \boldsymbol{E}+2 \boldsymbol{E} \times(\nabla \times \boldsymbol{E}) .
$$

Reconnections of optical C lines have recently been observed [30], and interpreted in terms of $\psi_{1}$.

\section{Concluding remarks}

The argument of section 2 can be translated into the language of singularity theory, by regarding the wavefunction as a map $\psi$ from $\mathrm{R}^{3}$ (i.e. $\boldsymbol{r}=\{x, y, z\}$ ) to $\mathrm{R}^{2}$ or $\mathrm{C}$, with a parameter $(t)$ inducing bifurcations. The $\Omega=0$ line is the singular set $\Sigma_{\psi}$, where the rank $D \psi$ is unity. Close to almost any intensity critical point on $\Sigma_{\psi}$, there is a local transformation [31] to the normal form

$$
\xi=t+x^{2} \pm y^{2}, \quad \eta=z,
$$

corresponding to a fold singularity; the $\Omega=0$ line is the $z$ axis, and the $+(-)$ sign corresponds to the loop (hyperbola reconnection) topology-changing events. The exceptions are points where $\Sigma_{\psi}$ is tangent to $\operatorname{ker}(D \psi)$; these are the points where $\boldsymbol{d} \cdot \boldsymbol{e}=0$, at which, assuming no further degeneracy, the normal form, replacing (33), can be chosen as

$$
\xi=t+x^{3}+y^{2}+x z, \quad \eta=u+z,
$$

involving an additional parameter $u$ : the image of the $\Omega=0$ line in the $\psi$ plane has a cusp (S) point at $\xi=t, \eta=u$. These normal forms do not capture all the three-dimensional geometry close to the topology-changing event, in particular the bending out of the plane embodied in equation (15).

How common are the topology-changing events that we have studied here? It is natural to investigate this by modelling $\psi$ as a complex Gaussian random wave, as we have done [23] for the density of vortex lines and other singularities. Although expressions can be found 
for statistics related to the topological events, analytical evaluation of the resulting multiple integrals has eluded us. Even the simpler associated problem of determining the spatial density of isolated critical points of intensity seems hard. It is important to understand the statistics of these events, because they organise the global topology of vortex lines in space (as in the analogous case of real wavefunctions in the plane, where avoided crossings of nodal lines influence the distribution of nodal domains [32]).

\section{Acknowledgment}

We thank Dr David Chillingworth for discussions about singularity theory. Our researches are supported by the Royal Society.

\section{Appendix. Solution of (17) for the $\Omega=0$ line}

Parallelism of $\nabla \xi$ and $\nabla \eta$ implies that (19) can be written

$$
a e_{z}+\operatorname{ImA} \cdot \boldsymbol{r}=\lambda \operatorname{Re} A \cdot r
$$

where $\lambda$ is a real parameter. The solution,

$$
\boldsymbol{r}(\lambda)=a(\lambda \operatorname{Re} \mathrm{A}-\operatorname{Im} \mathrm{A})^{-1} \cdot \boldsymbol{e}_{z},
$$

is the $\Omega=0$ line, parameterised by $\lambda$. Points near the origin correspond to large $\lambda$, so we can alternatively parameterise the line using $z$, using the identification

$$
z=a e_{z} \cdot(\lambda \operatorname{Re} \mathrm{A}-\operatorname{ImA})^{-1} \cdot e_{z} \approx \frac{a}{\lambda} e_{z} \cdot(\operatorname{ReA})^{-1} \cdot e_{z},
$$

whence (20) follows on replacing $\lambda$ by $z$ in the transverse $(\boldsymbol{R})$ components of (A2).

Although the form of the $\Omega=0$ line near the origin is sufficient for our study of the vortex interactions, it is interesting to explore (A2) in its generality. It is convenient to rewrite (A2) as

$$
\boldsymbol{r}(\lambda)=a(\operatorname{Re} \mathrm{A})^{-1 / 2}(\lambda \mathbf{1}-\mathrm{B})^{-1}(\operatorname{Re} \mathrm{A})^{-1 / 2} \cdot e_{z},
$$

where

$$
B=(\operatorname{Re} A)^{-1 / 2} \operatorname{Im} A(\operatorname{Re} A)^{-1 / 2} .
$$

We can write the $3 \times 3$ matrix B in terms of its eigenvalues $\lambda_{n}$ and eigenvectors $\boldsymbol{u}_{n}$, that is (in dyadic form)

$$
\mathrm{B}=\sum_{n=1}^{3} \lambda_{n} \boldsymbol{u}_{n} \boldsymbol{u}_{n}
$$

Thus (A2) becomes

$$
\boldsymbol{r}(\lambda)=a \sum_{n=1}^{3} \frac{\boldsymbol{u}_{n} \cdot(\operatorname{Re} \mathrm{A})^{-1 / 2} \cdot \boldsymbol{e}_{z}}{\lambda-\lambda_{n}}(\operatorname{ReA})^{-1 / 2} \cdot \boldsymbol{u}_{n}
$$

Globally, the $\Omega=0$ line for the quadratic model (12) has infinitely long segments, in each of which the asymptotes point in opposite directions and correspond to $\lambda=\lambda_{n}$. It follows from the symmetry of A that B is a symmetric matrix too; but B need not be real, because of the square roots in (A5) and the fact that Re A need not be positive-definite. Nevertheless, the characteristic equation for $B$ has real coefficients because det $B$ and $\operatorname{tr} B$ are real. Therefore either all three $\lambda_{n}$ are real, or one is real and the others form a complex-conjugate pair. In the 
first case, the $\Omega=0$ line consists of three separate segments, while in the second case there is just one segment.

From $\boldsymbol{r}(\lambda)$ it is easy to calculate the image curve $\{\xi(\boldsymbol{r}(\lambda)), \eta(\boldsymbol{r}(\lambda))\}$ in the $\psi$ plane. This possesses intensity critical points $\mathrm{P}$ closest to the origin $\mathrm{O}$ (figure 1), and cusp points $\mathrm{S}$ where the direction

$$
\boldsymbol{d}(\lambda) \propto \frac{\mathrm{d} \boldsymbol{r}(\lambda)}{\mathrm{d} \lambda}=a \sum_{n=1}^{3} \frac{\boldsymbol{u}_{n} \cdot(\operatorname{Re} \mathrm{A})^{-1 / 2} \cdot \boldsymbol{e}_{z}}{\left(\lambda_{n}-\lambda\right)^{2}}(\operatorname{Re} \mathrm{A})^{-1 / 2} \cdot \boldsymbol{u}_{n}
$$

is perpendicular to the common direction of $\nabla \xi$ and $\nabla \eta$, namely (cf (A1))

$$
e(\lambda) \propto \operatorname{Re} A \cdot r(\lambda) .
$$

\section{References}

[1] Nye J F and Berry M V 1974 Dislocations in wave trains Proc. R. Soc. Lond. A 336 165-90

[2] Nye J F 1999 Natural Focusing and Fine Structure of Light: Caustics and Wave Dislocations (Bristol: Institute of Physics Publishing)

[3] Vasnetsov M and Staliunas K 1999 Optical Vortices (Commack, New York: Nova Science Publishers)

[4] Berry M V, Dennis M R and Soskin M S 2004 The plurality of optical singularities (Editorial introduction to special issue) J. Opt. A: Pure Appl. Opt. 6 S155-156

[5] Feynman R P 1955 Application of Quantum Mechanics to Liquid Helium Progress in Low Temperature Physics vol 1 ed C J Gorter (Amsterdam: North-Holland) pp 17-53

[6] Pismen L M 1999 Vortices in Nonlinear Fields (Oxford: Oxford University Press)

[7] Berry M V and Dennis M R 2001 Knotted and linked phase singularities in monochromatic waves Proc. R. Soc. Lond. A 457 2251-63

[8] Berry M V and Dennis M R 2001 Knotting and unknotting of phase singularities in Helmholtz waves, paraxial waves and waves in 2+1 spacetime J. Phys. A: Math. Gen. 34 8877-88

[9] Leach J, Dennis M R, Courtial J and Padgett M J 2004 Knotted threads of darkness Nature 432165

[10] Samuels D C, Barenghi C F and Ricca R L 1998 Quantized vortex knots J. Low Temp. Phys. 110 509-14

[11] Dennis M R 2001 Topological Singularities in Wave Fields PhD Thesis University of Bristol

[12] Nye J F 2004 Local solutions for the interaction of wave dislocations J. Opt. A: Pure Appl. Opt. 6 S251-4

[13] Dennis M R 2003 Braided nodal lines in wave superpositions New J. Phys. 5134

[14] Nye J F 2006 Evolution of the hyperbolic umbilic diffraction pattern from Airy rings J. Opt. A: Pure Appl. Opt. 8 304-14

[15] Molina-Terriza G, Recolons J, Torres J P and Torner L 2001 Observation of the dynamical inversion of the topological charge of an optical vortex Phys. Rev. Lett. 87023902

[16] O'Holleran K, Padgett M J and Dennis M R 2006 Topology of optical vortex lines formed by the interference of three, four and five plane waves Opt. Exp. 14 3039-44

[17] Koplik J and Levine K 1993 Vortex reconnection in liquid helium Phys. Rev. Lett. 71 1375-8

[18] Bialynicki-Birula I, Bialynicka-Birula Z and Sliwa C 2000 Motion of vortex lines in quantum mechanics $P$ hys. Rev. A 61032110

[19] Nazarenko S and West R 2003 Analytical solution of nonlinear Schrödinger vortex reconnection J. Low Temp. Phys. 132 1-10

[20] Vilenkin A and Shellard E P S 2000 Cosmic Strings and Other Topological Defects (Cambridge: Cambridge University Press)

[21] Nye J F, Hajnal J V and Hannay J H 1988 Phase saddles and dislocations in two-dimensional waves such as the tides Proc. R. Soc. A 417 7-20

[22] Berry M V 1998 Much ado about nothing: optical dislocation lines (phase singularities, zeros, vortices ... ) Singular Optics (SPIE Proc. vol 3487) ed M S Soskin (Bellingham, WA: SPIE Optical Engineering Press) pp 1-5

[23] Berry M V and Dennis M R 2000 Phase singularities in isotropic random waves Proc. R. Soc. Lond. A 456 2059-79

[24] Berry M V 2005 Phase vortex spirals J. Phys. A: Math. Gen. L745-51

[25] Nye J F and Hajnal J V 1987 The wave structure of monochromatic electromagnetic radiation Proc. R. Soc. A $40921-36$ 
[26] Berry M V and Dennis M R 2001 Polarization singularities in isotropic random vector waves Proc. R. Soc. A 457 141-55

[27] Dennis M R 2004 Local phase structure of wave dislocation lines: twist and twirl J. Opt. A: Pure Appl. Opt. $6 \mathrm{~S} 202-8$

[28] Gibbs J W 1928 Elements of vector analysis (Collected Works, Volume II Part 2) (London: Longmans Green)

[29] Berry M V 2004 Index formulae for singular lines of polarization J. Opt. A: Pure Appl. Opt. 6 675-8

[30] Flossmann F, Schwarz U T, Maier M and Dennis M R 2005 Polarization singularities from unfolding an optical vortex through a birefringent crystal Phys. Rev. Lett. 95253901

[31] Gibson C G 1979 Singular Points of Smooth Mappings (Boston, MA: Pitman)

[32] Monastra A G, Gnutzmann S and Smilansky U 2003 Avoided intersections of nodal lines J. Phys. A: Math. Gen. 36 1845-53 